\title{
Probabilistic Locational Marginal Price by Monte Carlo Simulation with Latin Hypercube Sampling and Scenario Reduction Techniques
}

\author{
Pham Nang Van \\ Department of Electric Power Systems, Hanoi University of Science and Technology, Hanoi, Vietnam \\ Email:van.phamnang@hust.edu.vn
}

\begin{abstract}
Leveraging Monte Carlo Simulation (MCS) combined with simple random sampling (SRS) to evaluate probabilistic locational marginal price (P-LMP) requires long computation time and large computer storage. The paper proposes the joint usage of Latin hypercube sampling (LHS) with sample reduction techniques called the fast forward selection (FFS) algorithm into Monte Carlo simulation for calculation of the P-LMP. This fast forward selection algorithm is needed to cut down the number of samples while keeping most of the stochastic information embedded in such samples. The LHS-FFS-based P-LMP is investigated using IEEE 6-bus and 24-bus systems. This method is compared with SRS and LHS only. The LHS-FFS approach is found to be efficient and flexible; therefore, it has the potential to be applied in many power system probabilistic problems.
\end{abstract}

Keywords: Latin hypercube sampling (LHS), Monte Carlo simulation (MCS), probabilistic locational marginal price (P-LMP), fast forward selection (FFS) algorithm, uncertainty.

\section{Introduction}

Currently, many countries around the world, including Vietnam, have been operating wholesale electricity markets. In the wholesale electricity market, the market participants are generation companies (GENCOS) and distribution companies (DISCOS). The market operator collects generating offers by producers, load bids by consumers and clears the market by maximizing social welfare. To make payments in the electricity market, locational marginal price (LMP) is calculated. The difference in LMPs between two nodes of a branch is due to congestion and losses on that branch. The locational marginal pricing methodology is widely used in electricity markets to determine the electricity prices and evaluate the transmission congestion cost.

Furthermore, the issue of climate change has required the pressing need for limiting industrial emissions of greenhouse gases. In addition to the tragic consequences of climate change, there is an energy crisis in many countries in the world due to the depletion of fossil fuels. Therefore, renewable energy has been prominent in most industrialized countries with the aim of decarbonizing in the electricity sector as well as meeting the rising demand for energy and safeguarding the security of the energy supply. Solar energy, wind, geothermal, biomass, waves and hydrogen energy are major renewables. Wind power is an economically attractive renewable for producing electricity. Therefore, wind power is

ISSN: 2734-9373

https://doi.org/10.51316/jst.150.ssad.2021.31.1.6 Received: 28 September 2018; accepted: 21 February 2021 being integrated with higher and higher amounts into electric energy systems throughout the world [1]. However, wind power is also an intermittent source. The integration of a significant amount of wind power into a power system results in critical operational challenges, which, in turn, originate alternations in locational marginal prices (LMPs) [2].

LMP is the additional cost when the load increases at a specific node. The LMP-based approach has been dominant to determine electricity prices and manage transmission congestion in power markets. Locational marginal prices may encompass three components: marginal energy price, marginal congestion price, and marginal loss price. The optimal power flow (OPF) has been applied in the power industry to calculate LMP [3].

The uncertainty and variability of wind generation could lead to LMP variations [4]. Evaluating the impacts of the uncertain parameters on LMP is of most importance in power system planning and operation. Different techniques, such as the probabilistic approach [5-6], possibilistic method [7], hybrid possibilistic-probabilistic strategies [8], information gap decision theory [9], and robust optimization [4], have been developed to deal with uncertainties. Among these methods, probabilistic techniques are more appropriate for the impact assessment of renewable energy sources [10].

Different approaches have been introduced to determine the probabilistic locational marginal price (P-LMP) from solving the probabilistic optimal power flow (P-OPF). In Monte Carlo (MC) based techniques, samples from random input variables are 
generated, and then the deterministic problem is solved for each sample [6]. Simple random sampling (SRS) is one of the most popular MC techniques in which samples are randomly generated from input variables' distribution functions. While the SRS can provide highly accurate results, it suffers from the drawback of heavy computation time and the high storage required for many repeated calculations. The P-OPF problem can be solved by employing the Latin hypercube sampling (LHS) approach [6]. Although the number of Latin hypercube samples is reduced compared with the simple random samples, the number of these samples is generally large to represent the uncertainty involved accurately. This may render the P-OPF problem intractable for electricity market operation [11]. As a result, a scenario reduction is applied to decrease an initial LHS sample size. There are several methods available to reduce scenarios to be used in the P-OPF problem [12]. These methods seek to obtain a reduced number of scenarios that best retain the essential features of a given original scenario set according to a probability distance. In [13], a scenario reduction algorithm based on submodular function optimization is employed to optimize the number of scenarios and rank these scenarios.

In this paper, P-LMP is calculated using MC with LHS combined with scenario reduction techniques, namely Fast Forward Selection (FFS) Algorithm. This paper's main contribution is that PLMP determined using three different approaches, particularly simple random sampling, Latin hypercube sampling, and Latin hypercube sampling combined scenario reduction techniques, are compared and analyzed.

The paper's remainder is presented as follows: Section 2 presents Latin hypercube sampling; Section 3 presents the FFS sample reduction technique. The LMP calculation method based on the ACOPF market-clearing model is presented in Section 4. Section 5 describes the MC simulation procedure for calculating the probabilistic locational marginal price. The calculation examples and the comparisons of different P-LMP approaches are presented in Section 6. The conclusion is given in Section 7.

\section{Latin Hypercube Sampling}

Latin hypercube sampling is a recent development in sampling technology designed to accurately reflect the input distribution through sampling in fewer iterations when compared with the simple random sampling method. The key to Latin hypercube sampling is the stratification of the input probability distributions [5]. Stratification divides the cumulative curve into equal intervals on the cumulative probability scale (0 to 1.0$)$. A sample is then randomly taken from each interval of the input distribution. Sampling is forced to represent values in each interval and is forced to recreate the input probability distribution. With Latin hypercube, a sample is drawn from each interval; therefore, the samples more accurately reflect the distribution of values in the input probability distribution.

It is assumed that there are the $K$ input random variables in a probabilistic formulation, including $X_{1}$, $X_{2}, \ldots, X_{K}$. The cumulative distribution function of $X_{k}$ is expressed as follows:

$$
Y_{k}=F_{k}\left(X_{k}\right) \quad \forall k=1,2, \ldots, K
$$

If the sample size is $N$, then the range of $Y_{k}$ is separated into $N$ intervals that are not overlapping. Each interval has a length of $1 / N$. One sampling value of $Y_{k}$ is selected from the midpoint or random point of each interval. After that, the inverse function of the cumulative distribution function is employed to determine the sampling values of $X_{k}$.

As illustrated in Fig. 1, if the midpoint of each interval is adopted, the $n$th sample of $X_{k}$ can be calculated as follows:

$$
x_{k n}=F_{k}^{-1}\left(\frac{n-0.5}{N}\right) \quad \forall k=1,2, \ldots, K
$$

Then, a row of the sampling matrix $\left[x_{k 1} \ldots x_{k n} \ldots x_{k N}\right]$ is formed from the sample values of $X_{k}$. When all the $K$ input random variables are sampled, a sampling matrix $\mathbf{X}(K \times N)$ can be constructed.

In this paper, Latin hypercube samples are generated using MATLAB software [14].

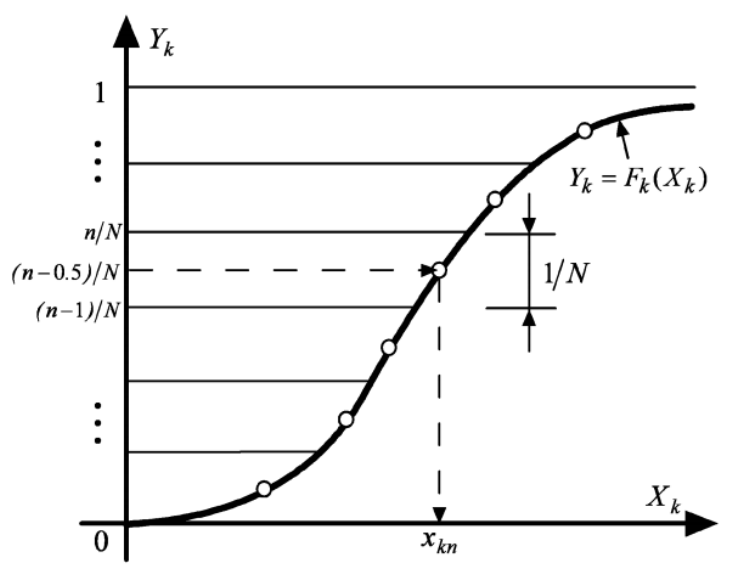

Fig. 1. Latin hypercube sampling technology

\section{Sample reduction technique}

\subsection{Algorithm}

A scenario reduction methodology seeks to downsize a scenario set while still keeping as intact as possible the stochastic information embedded in it. Next, we briefly explain a scenario reduction procedure, which relies on the concept of probability 
distance, namely the Fast Forward Selection (FFS) Algorithm.

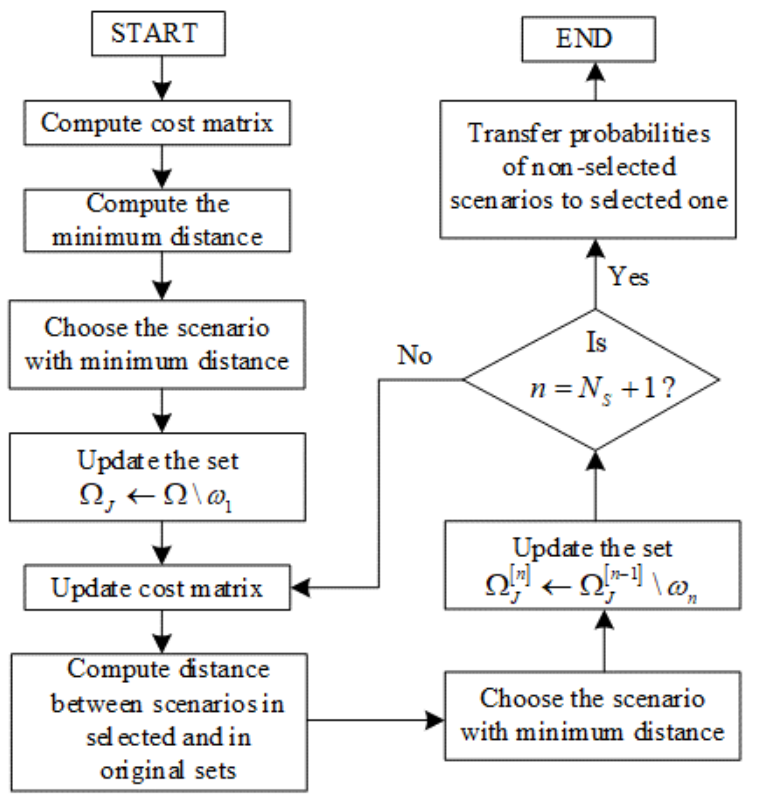

Fig. 2. Flow diagram of the Fast Forward Selection Algorithm to reduce scenario

The FFS algorithm is an iterative greedy process starting with an empty set. In each iteration, from the set of non-selected scenarios, the scenario which minimizes the Kantorovich distance between the reduced and original sets is selected. Then, this scenario is included in the reduced set. The algorithm stops if either a specified number of scenarios or a certain Kantorovich distance is attained.

The Kantorovich distance can be equivalently determined as:

$$
D_{K}\left(Q, Q^{\prime}\right)=\sum_{\omega \in \Omega / \Omega_{S}} \pi_{\omega} \min _{\omega^{\prime} \in \Omega_{S}} v\left(\omega, \omega^{\prime}\right)
$$

where $v\left(\omega, \omega^{\prime}\right)$ is a cost function, which is the vector distance between scenario $\omega$ and $\omega^{\prime} ; Q$ and $Q^{\prime}$ are the probability distributions in the initial scenario set $\Omega$ and selected scenario set $\Omega_{\mathrm{S}}$, respectively; $\pi_{\omega}$ is the probability of scenario $\omega$.

FFS algorithm is depicted in Fig. 2. A step-bystep explanation of the algorithm [15]-[16] is also provided in the following:

Step 0

Compute cost function $v^{[1]}\left(\omega, \omega^{\prime}\right)$ for each pair scenarios $\omega$ and $\omega^{\prime}$.

Step 1

Compute $d_{\omega}=\sum_{\omega=1}^{N_{\Omega}} \pi_{\omega} v^{[1]}\left(\omega, \omega^{\prime}\right) \quad \forall \omega \in \Omega$
Choose $\omega_{1} \in \arg \min _{\omega \in \Omega} d_{\omega}$

Update the set $\Omega_{J} \leftarrow \Omega \backslash \omega_{1}$

Step $n$

Compute $v^{[n]}\left(\omega, \omega^{\prime}\right)$ where

$v^{[n]}\left(\omega, \omega^{\prime}\right)=$

$=\min v^{[n-1]}\left(\omega, \omega^{\prime}\right), v^{[n-1]}\left(\omega, \omega_{n-1}\right) \forall \omega, \omega^{\prime} \in \Omega_{J}^{[n-1]}$

$d_{\omega}^{[n]}=\sum_{\omega \in \Omega_{J}^{[n-1]} \backslash \omega} \pi_{\omega^{\prime}} \nu^{[n]}\left(\omega^{\prime}, \omega\right) \quad \forall \omega \in \Omega_{J}^{[n-1]}$

Choose $\omega_{n} \in \arg \min d_{\omega}^{n}$

Update the set $\Omega_{J}^{[n]} \leftarrow \Omega_{J}^{[n-1]} \backslash \omega_{n}$

$$
\begin{aligned}
\text { Step } & N_{S}+1 \\
& \Omega_{J}^{*}=\Omega_{J}^{N_{\Omega_{J}}} ; \Omega_{S}^{*}=\Omega \backslash \Omega_{J}^{*} \\
& \pi_{\omega}^{*}=\pi_{\omega}+\sum_{\omega^{\prime} \in J(\omega)} \pi_{\omega^{\prime}}
\end{aligned}
$$

where

$$
\begin{aligned}
& J(\omega)=\omega^{\prime} \in \Omega_{J}^{*} \mid \omega=j\left(\omega^{\prime}\right) \text { such that } \\
& j\left(\omega^{\prime}\right)=\arg \min _{\omega^{\prime \prime} \in \Omega_{S}^{*}} v\left(\omega^{\prime \prime}, \omega^{\prime}\right)
\end{aligned}
$$

where $\Omega_{J}^{*}$ is the final set of deleted scenarios and $\Omega_{S}^{*}$ is the set of selected scenarios after the scenarioreduction process.

\subsection{Illustrative Example}

It is assumed that the four power scenarios $P_{\omega}, \omega=1, \ldots, 4$ with associated probabilities $\pi_{\omega}$ can statistically represent the generating output of a 100 MW wind farm in a given period.

Table 1. Four power scenarios of illustrative example

\begin{tabular}{|c|c|c|c|c|}
\hline Scenario & 1 & 2 & 3 & 4 \\
\hline$P_{\omega}(\mathrm{MW})$ & 5 & 40 & 60 & 100 \\
\hline$\pi_{\omega}$ & 0.25 & 0.2 & 0.2 & 0.35 \\
\hline
\end{tabular}

Suppose that the number of wind power scenarios being reduced is equal to 2 . The fast forward selection algorithm works as follows:

Step 0: Calculate the cost function

$$
v\left(\omega, \omega^{\prime}\right)=\left\|P_{\omega}^{\mathrm{W}}-P_{\omega^{\prime}}^{\mathrm{W}}\right\|, \forall \omega, \omega^{\prime} \in \Omega
$$

where $\Omega=\{1,2,3,4\}$

The values of function $v$ can be cleverly organized into a symmetric matrix whose diagonal elements equal zero: 


$$
v=\left(\begin{array}{cccc}
0 & 35 & 55 & 95 \\
35 & 0 & 20 & 60 \\
55 & 20 & 0 & 40 \\
95 & 60 & 40 & 0
\end{array}\right) \mathrm{MW}
$$

Step 1: Scenario $\omega$ that minimizes the resulting Kantorovich distance between the reduced and original sets is chosen:

$$
\begin{aligned}
& d_{1}=\pi_{2} v(1,2)+\pi_{3} v(1,3)+\pi_{4} v(1,4)=51,25 \mathrm{MW} \\
& d_{2}=\pi_{1} v(2,1)+\pi_{3} v(2,3)+\pi_{4} v(2,4)=33,75 \mathrm{MW} \\
& d_{3}=\pi_{1} v(3,1)+\pi_{2} v(3,2)+\pi_{4} v(3,4)=31,75 \mathrm{MW} \\
& d_{4}=\pi_{1} v(4,1)+\pi_{2} v(4,2)+\pi_{3} v(4,3)=43,75 \mathrm{MW}
\end{aligned}
$$

Therefore,

$$
\begin{aligned}
& \Omega_{S}^{[1]}=\{3\}, \\
& \Omega_{J}^{[1]}=\{1,2,4\}
\end{aligned}
$$

Step 2: The cost matrix is updated as follows:

$$
\begin{aligned}
& v^{[2]}(1,2)=\min \{v(1,2), v(1,3)\}=35 \mathrm{MW} \\
& v^{[2]}(1,4)=\min \{v(1,4), v(1,3)\}=55 \mathrm{MW} \\
& v^{[2]}(2,1)=\min \{v(2,1), v(2,3)\}=20 \mathrm{MW} \\
& v^{[2]}(2,4)=\min \{v(2,4), v(2,3)\}=20 \mathrm{MW} \\
& v^{[2]}(4,1)=\min \{v(4,1), v(4,3)\}=40 \mathrm{MW} \\
& v^{[2]}(4,2)=\min \{v(4,2), v(4,3)\}=40 \mathrm{MW}
\end{aligned}
$$

Consequently,

$$
v^{[2]}=\left(\begin{array}{cccc}
0 & 35 & 55 & 55 \\
20 & 0 & 20 & 20 \\
55 & 20 & 0 & 40 \\
40 & 40 & 40 & 0
\end{array}\right) \mathrm{MW}
$$

Considering the new cost matrix $v^{[2]}$, the scenario $\omega$ selected from $\Omega_{J}^{[1]}$ is the one that minimizes the Kantorovich distance between the subsequent reduced set $\Omega_{S}^{[2]}$ and the original set $\Omega$ :

$$
\begin{aligned}
& d_{1}^{[2]}=\pi_{2} v^{[2]}(2,1)+\pi_{4} v^{[2]}(4,1)=18 \mathrm{MW} \\
& d_{2}^{[2]}=\pi_{1} v^{[2]}(1,2)+\pi_{4} v^{[2]}(4,2)=22,75 \mathrm{MW} \\
& d_{4}^{[2]}=\pi_{1} v^{[2]}(1,4)+\pi_{2} v^{[2]}(2,4)=17,75 \mathrm{MW}
\end{aligned}
$$

Hence,

$$
\begin{aligned}
& \Omega_{S}^{[2]}=\Omega_{S}^{*}=\{3,4\} \\
& \Omega_{J}^{[2]}=\Omega_{J}^{*}=\{1,2\}
\end{aligned}
$$

Step 3: The scenario reduction algorithm stops with the optimal transfer of probabilities from the set of non-selected scenarios $\Omega_{J}^{*}$ to selected ones $\Omega_{S}^{*}$.

\section{Given that:}

Scenario 3 in $\Omega_{S}^{*}$ is the closest one to scenario 1 in $\Omega_{J}^{*}(v(1,3)=55$ while $v(1,4)=95)$ and

Scenario 3 in $\Omega_{S}^{*}$ is the closest one to scenario 2 in $\Omega_{J}^{*}(v(2,3)=20$ while $v(2,4)=60)$.

It follows:

$$
\begin{aligned}
& \pi_{3}^{*}=\pi_{3}+\pi_{2}+\pi_{1}=0,65 \\
& \pi_{4}^{*}=\pi_{4}=0,35
\end{aligned}
$$

To sum up, a reduced scenario set $\Omega_{S}^{*}=\{3,4\}$ with associated probabilities $\pi_{3}^{*}=0,65$ and $\pi_{4}^{*}=0,35$ is provided.

\section{Market-Clearing Model}

The clearing of an electricity market involves two primary tasks, namely, determining the production (consumption) level of every producer (consumer) and settling the locational marginal price (LMP) at which every producer (consumer) is paid (charged) for its energy production (consumption). In this paper, we assume that an independent system operator (ISO) is in charge of conducting these tasks by solving the following problem:

\subsection{Objective Function}

The objective of the market-clearing problem is to maximize the total social welfare (SW), as shown in Equation (2) below:

$\operatorname{Maximize} \mathrm{SW}=\sum_{j=1}^{N_{D}} \sum_{k=1}^{N_{D j}} \lambda_{D j k} \cdot P_{D j k}-\sum_{i=1}^{N_{G}} \sum_{b=1}^{N_{G i}} \lambda_{G i b} \cdot P_{G i b}$

where $\lambda_{\text {Gib }}$ is the price of the energy block $b$ offered by generating unit $i, P_{G i b}$ is the power of the energy block $b$ offered by generating unit $i, \lambda_{D j k}$ is the price of the energy block $k$ bid by demand $j, P_{D j k}$ is the power block $k$ bid by demand $j$.

\subsection{Constraints}

\subsubsection{Power balance}

The active power and reactive power injected into bus $i$ is subjected to the following power flow equations:

$$
\begin{aligned}
P_{i} & =P_{G i}+P_{W i}-P_{D i} \\
& =\left|\dot{U}_{i}\right| \sum_{j=1}^{n}\left|\dot{U}_{j}\right|\left(G_{i j} \cos \delta_{i j}+B_{i j} \sin \delta_{i j}\right) \\
Q_{i} & =Q_{G i}+Q_{W i}-Q_{D i} \\
& =\left|\dot{U}_{i}\right| \sum_{j=1}^{n}\left|\dot{U}_{j}\right|\left(G_{i j} \sin \delta_{i j}-B_{i j} \cos \delta_{i j}\right)
\end{aligned}
$$


where $P_{G i}, Q_{G i}$ are real and reactive power generated by the conventional generators at bus $i$, respectively; $P_{W i}, Q_{W i}$ are real and reactive power generated by the wind turbine at bus $i$, respectively; $P_{D i}, Q_{D i}$ are the active and reactive power of demand at node $i$, respectively; $\left|\dot{U}_{i}\right|$ and $\left|\dot{U}_{j}\right|$ are the magnitudes of voltage at node $i$ and $j$, respectively; $G_{i j}$ and $B_{i j}$ are the real and imaginary part of element $i j$ of the admittance matrix, respectively; $\delta_{i j}$ is the voltage angle difference between nodes $i$ and $j ; n$ is the number of buses in power systems.

4.2.2 The active power limit of each generation block of conventional producer

$$
0 \leq P_{\text {Gib }} \leq P_{\text {Gib }}^{\max }(\forall i, b)
$$

where $P_{G i b}^{\max }$ is the MW size of block $b$ offered by generating unit $i$.

\subsubsection{Power limit of the generating units}

For a generating unit, its active power is subjected to the constraint (5), as follows:

$$
\begin{aligned}
& P_{G i}^{\min } \leq P_{G i} \leq P_{G i}^{\max }(\forall i) \\
& 0 \leq P_{W q} \leq P_{W q}^{\max }(\forall q)
\end{aligned}
$$

\subsubsection{Limits on the price-sensitive loads}

In a wholesale power market, the loads are considered to consist of two components: fixed load and price-sensitive load. The demand curve of pricesensitive loads can consist of several blocks, each with a lower and an upper limit, as shown in (6)-(7).

$$
\begin{aligned}
& P_{D j}^{\mathrm{E} \min } \leq P_{D j}^{E} \leq P_{D j}^{\mathrm{E} \max }(\forall j) \\
& 0 \leq P_{D j k}^{E} \leq P_{D j k}^{\mathrm{E} \max }(\forall j, \mathrm{k})
\end{aligned}
$$

where $P_{D j}^{E}$ is elastic power of demand $j$ and $P_{D j k}^{\mathrm{Emax}}$ is the MW size of block $k$ bid by demand $j$.

\subsubsection{Branch flow limits}

The branch flow can be expressed by a function of injected active power via the power distribution factors [3]

$$
-P_{k}^{\max } \leq P_{k}=\sum_{i=1}^{n} G S F_{k-i}\left(P_{G i}+P_{W i}-P_{D i}\right) \leq P_{k}^{\max }
$$

where $G S F_{k-i}$ is the sensitivity of branch power flow $k$ with respect to injected power $i$ and $P_{k}^{\max }$ is the power flow limit on branch $k$.

Sensitivity of branch power flow $k$ with respect to injected power $i$ is defined as a power flow increase on the $k$ th line when the power injected in $i$ th node increases by $1 \mathrm{MW}$ and is computed as follows:

$$
G S F_{k-i}=\frac{\Delta P_{k}^{i}}{\Delta P_{i}}
$$

The $G S F_{k-i}$ above depends solely on the structure of electrical networks; therefore, these factors can be calculated offline using sparse matrix techniques. However, the $G S F_{k-i}$ depends on the location of the voltage reference node.

Note that wind production is treated as a negative demand that can be spilled. This is the case in which wind power is treated in most energy systems worldwide. Moreover, this is equivalent to assuming that wind producers offer their energy production at zero price and are not penalized in the real-time market for their energy imbalances.

\subsection{LMP Calculation and Components}

The locational marginal price (LMP) of electricity at a location is defined as the least cost to supply the next incremental of demand at that location consistent with all power system operating constraints. The active power LMP at each bus $i$ is simply the Lagrange multiplier related to that bus's real power balance constraint.

The locational marginal price consists of the following components [4]:

$$
L M P_{i}=L M P_{E}-L F_{i} \cdot L M P_{E}+\sum_{k} G S F_{k-i} \cdot \mu_{k}
$$

where $L M P_{E}$ is the marginal energy price, $L F_{i}$ is the loss factor for node $i, \mu_{k}$ is the shadow price of transmission constraint on the $k$ th line.

The loss factor can be computed as follows:

$$
\begin{gathered}
P_{\text {loss }}=\sum_{k=1}^{M} P_{k}^{2} R_{k} \\
\frac{\partial P_{\text {loss }}}{\partial P_{i}}=\frac{\partial}{\partial P_{i}}\left(\sum_{k=1}^{M} P_{k}^{2} R_{k}\right) \\
P_{k}=\sum_{i=1}^{n} G S F_{k-i}\left(P_{G i}+P_{W i}-P_{D i}\right)=\sum_{i=1}^{n} G S F_{k-i} \times P_{i}
\end{gathered}
$$

where $R_{k}$ is the resistance of line $k, P_{\text {loss }}$ is the total loss of the power system, and $M$ is the number of lines. follows:

Equation (12) can be expanded further as

$$
\begin{aligned}
\frac{\partial P_{\text {loss }}}{\partial P_{i}} & =\sum_{k=1}^{M} \frac{\partial}{\partial P_{i}}\left(P_{k}^{2} R_{k}\right) \\
& =\sum_{k=1}^{M} R_{k} \times 2 P_{k} \times \frac{\partial P_{k}}{\partial P_{i}} \\
& =\sum_{k=1}^{M} 2 \times R_{k} \times G S F_{k-i} \times\left(\sum_{i=1}^{n} G S F_{k-i}\left(P_{G i}+P_{W i}-P_{D i}\right)\right)
\end{aligned}
$$


The market-clearing model (2)-(8) is a nonlinear optimization problem. A great variety of optimization techniques can be used to solve this problem, including the generalized reduced gradient [17], Newton's method [18], sequential linear programming [19], and more recently, interior point methods [20].

\subsection{Sequential Linear Programming (SLP)}

This subsection describes the sequential linear programming method to deal with the abovementioned optimization problem, which is implemented in POWERWORLD software. The solution procedure consists of the following steps:

Step 1: Select the set of initial control variables.

Step 2: Solve the power flow problem to obtain a feasible solution that satisfies the power balance equality constraints.

Step 3: Linearize the objective function and inequality constraints around the power flow solution and formulate the linear programming (LP) problem.

Step 4: Solve the LP problem and attain optimal incremental control variables $\Delta P_{G i}$

Step 5: Update the control variables

$$
P_{G i}^{(k+1)}=P_{G i}^{(k)}+\Delta P_{G i}
$$

Step 6: Obtain the power flow solution with updated control variables.

Step 7: Check the convergence. If $\Delta P_{G i}$ in step 4 are below the user-defined tolerance, the solution converges. Otherwise, go to step 3.

\section{Probabilistic Locational Marginal Price (P- LMP)}

\subsection{Wind Farm Distribution}

According to the significant amount of data collected from wind farms, the relationship between the output power of wind turbine generators and the wind speed is commonly expressed as:

$$
P_{W}= \begin{cases}0 & v>v_{\text {out }} \quad \text { or } \quad v<v_{\text {in }} \\ P_{W r} \frac{v-v_{\text {in }}}{v_{r}-v_{\text {in }}} & v_{\text {in }} \leq v \leq v_{r} \\ P_{W r} & v_{r} \leq v \leq v_{\text {out }}\end{cases}
$$

where $P_{W r}$ is the rated power of wind turbine, $v$ is wind speed, $v_{\text {in }}$ is cut-in wind speed, $v_{\text {out }}$ is cut-out wind speed, $v_{r}$ is the rated wind speed.

The probability density function (PDF) of the wind speed can be described accurately by the Weibull distribution [21]:

$$
f_{v}(v)= \begin{cases}0 & v<0 \\ \frac{k}{c}\left(\frac{v}{c}\right)^{k-1} \exp \left[-\left(\frac{v}{c}\right)^{k}\right] & v \geq 0\end{cases}
$$

where $k$ and $c$ is the shape and scale factor of Weibull distribution, respectively.

\subsection{Simulation Procedure}

LMP random sequences for forecasted wind speed with Weibull probability density function (PDF) are generated using Monte Carlo simulation. Monte Carlo simulation is based on simulating the value of uncertain parameters using random number generators to determine the value of the sampled statuses and estimating the outputs. After a large number of simulations are implemented, it is expected that the probability distribution of the outputs will be obtained with a degree of confidence to the confidence interval.

To produce a non-uniform probability distribution sequence, we first generate a uniform distribution sequence and then use mathematical tools to transform this sequence into the Weibull distribution sequence. Steps of solving probabilistic LMP are given as follows:

- Construct a probability model for wind speed, according to (16).

- Generate Latin hypercube random sequences of wind speed using the constructed models in step 1, according to Section 2. In this study, the number of Latin hypercube samples are 500 and 1000 , respectively.

Compute Latin hypercube random sequences of wind power using Equation (15) and random wind speed sequences in step 2 .

- $\quad$ Reduce the sample number of wind power using the FFS technique, as manifested in Section 3. In this paper, the Latin hypercube samples after scenario reduction implementation are 100 . These reduced Latin hypercube samples are used as inputs for the market-clearing model described in Section 4.

- Solve the market-clearing model for each Latin hypercube sample by leveraging POWERWORLD software [22] and obtain the Locational Marginal Prices.

- Perform statistical analysis to obtain the statistical property of the P-LMP, such as PDF and mean value, after solving $N$ problems for market-clearing.

The Monte Carlo simulation will stop when a predefined convergence threshold $\varepsilon$ has been reached. The stopping criterion is mathematically shown as follows: 
JST: Smart Systems and Devices

Volume 31, Issue 1, May 2021, 041-049

$$
\frac{\sigma[E(L M P)]}{E(L M P)} \leq \varepsilon
$$

where $E(L M P)$ is the mean value of LMP, $\sigma$ is the variance of a random variable. The mean value is determined as the following expressions:

$$
E(L M P)=\frac{\sum_{k=1}^{N} L M P_{k}}{N}
$$

where $N$ is the number of samples.

\section{Case Studies}

A series of P-OPF studies with SRS, LHS, and LHS-FFS are carried out on 6-bus and IEEE 24-bus test systems, respectively.

\subsection{IEEE 6-Bus System}

A six-bus system is considered, which is shown in Fig.3. The branch data and bus data are presented in Table 1 and Table 2, respectively. Generator offers are provided in Table 3.

To parameters of the system as per unit, the power base of the six-bus system is set at 100 MVA. The voltage base of this system is set at $230 \mathrm{kV}$.

A wind farm is assumed to have been constructed at bus 6 . This wind farm consists of 2.5 MW wind generators, model Nordex N80/2500 with a hub height of $105 \mathrm{~m}$. The power curve of this turbine model is supplied by the Danish Wind Industrial Association [21]. The Weibull distribution with shape parameter $(k)$, and scale parameter $(c)$ equal to 1.6 and 9.7, respectively, is deployed to model wind speed.

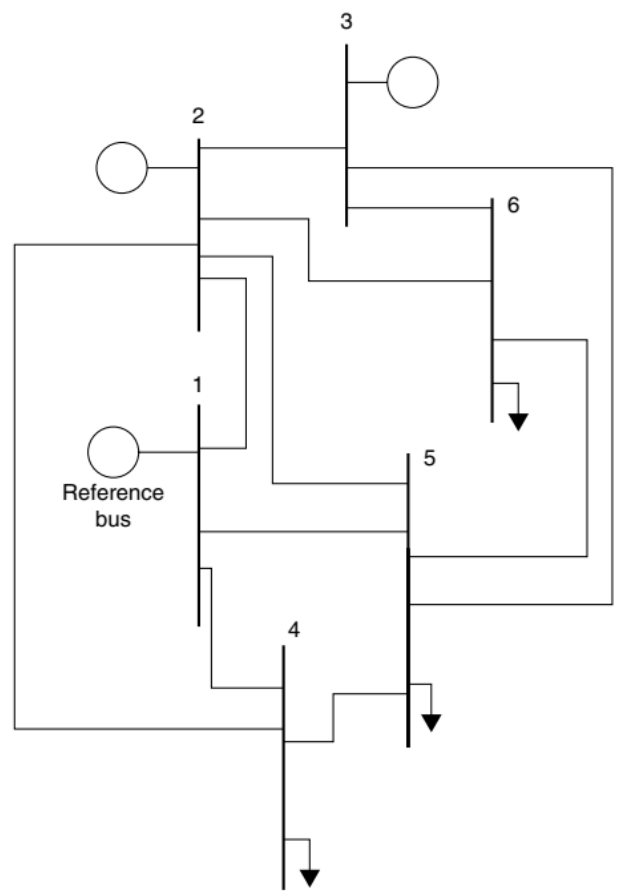

Fig. 3. Six-bus system topology
Table 1. Line data for 6-bus system

\begin{tabular}{|c|c|c|c|c|c|}
\hline From & To & $R(\mathrm{pu})$ & $X(\mathrm{pu})$ & $B(\mathrm{pu})$ & Rate (MVA) \\
\hline 1 & 2 & 0.1 & 0.2 & 0.04 & 100 \\
\hline 1 & 4 & 0.05 & 0.2 & 0.04 & 100 \\
\hline 1 & 5 & 0.08 & 0.3 & 0.06 & 100 \\
\hline 2 & 3 & 0.05 & 0.25 & 0.06 & 60 \\
\hline 2 & 4 & 0.05 & 0.1 & 0.02 & 60 \\
\hline 2 & 5 & 0.1 & 0.3 & 0.04 & 60 \\
\hline 2 & 6 & 0.07 & 0.2 & 0.05 & 60 \\
\hline 3 & 5 & 0.12 & 0.26 & 0.05 & 60 \\
\hline 3 & 6 & 0.02 & 0.1 & 0.02 & 60 \\
\hline 4 & 5 & 0.2 & 0.4 & 0.08 & 60 \\
\hline 5 & 6 & 0.1 & 0.3 & 0.06 & 60 \\
\hline
\end{tabular}

Table 2. Bus data for 6-bus system

\begin{tabular}{|c|c|c|c|c|c|}
\hline Number & Type & $\begin{array}{c}P_{\mathrm{D}} \\
(\mathrm{MW})\end{array}$ & $\begin{array}{c}Q_{\mathrm{D}} \\
(\mathrm{MVAr})\end{array}$ & $\begin{array}{c}P_{G}^{\max } \\
(\mathrm{MW})\end{array}$ & $\begin{array}{c}P_{G}^{\min } \\
(\mathrm{MW})\end{array}$ \\
\hline 1 & 3 & & & 200 & 50 \\
\hline 2 & 2 & & & 150 & 37.5 \\
\hline 3 & 2 & & & 180 & 45 \\
\hline 4 & 1 & 100 & 15 & & \\
\hline 5 & 1 & 100 & 15 & & \\
\hline 6 & 1 & 100 & 15 & & \\
\hline
\end{tabular}

Type 3 = swing bus, type 2 = generator bus, type 1 = load bus

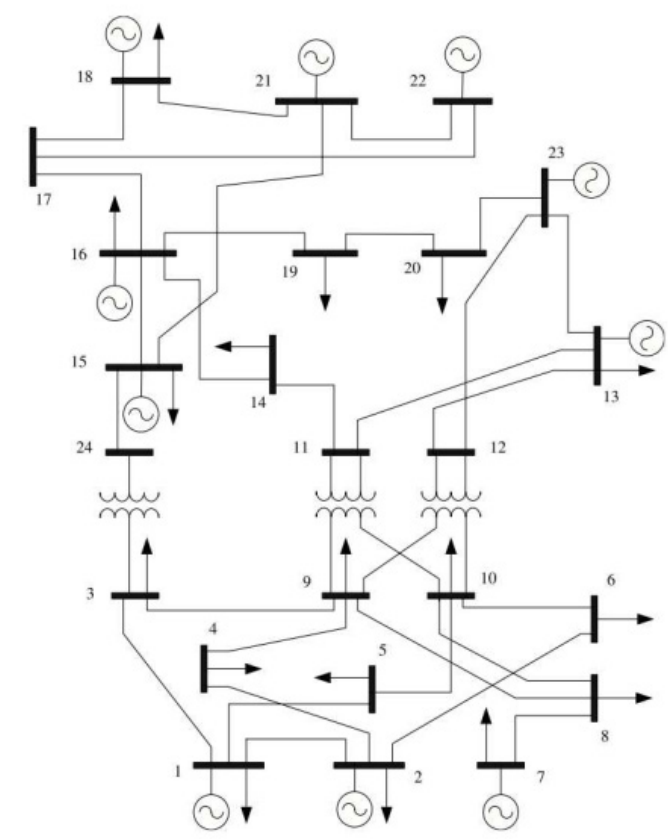

Fig. 4. 24-bus power system 
JST: Smart Systems and Devices

Volume 31, Issue 1, May 2021, 041-049

Table 3. Generator offers (VND/kWh) for 6-bus system

\begin{tabular}{|c|c|c|c|c|c|c|c|c|c|c|c|c|c|c|c|}
\hline & \multicolumn{5}{|c|}{ Generator 1} & \multicolumn{5}{c|}{ Generator 2} & \multicolumn{5}{c|}{ Generator 3} \\
\hline Block & 1 & 2 & 3 & 4 & 5 & 1 & 2 & 3 & 4 & 5 & 1 & 2 & 3 & 4 & 5 \\
\hline $\begin{array}{c}\text { Power } \\
(\mathrm{MW})\end{array}$ & 50 & 50 & 50 & 30 & 20 & 37.5 & 30 & 30 & 20 & 32.5 & 45 & 40 & 30 & 25 & 40 \\
\hline $\begin{array}{c}\text { Price } \\
(\mathrm{d} / \mathrm{kWh})\end{array}$ & 500 & 800 & 1200 & 1500 & 2000 & 200 & 800 & 1200 & 1600 & 2500 & 600 & 1000 & 1300 & 1800 & 2300 \\
\hline
\end{tabular}

Table 4. The mean LMP (VND/kWh) of six-bus system

\begin{tabular}{|l|c|c|c|c|c|c|}
\hline Bus & 1 & 2 & 3 & 4 & \multicolumn{1}{c|}{5} & 6 \\
\hline SRS (5000 samples) & 1196.898 & 1214.634 & 1217.834 & 1269.807 & 1278.744 & 1249.164 \\
\hline LHS (1000 samples) & 1196.762 & 1214.38 & 1217.388 & 1269.585 & 1278.411 & 1248.612 \\
\hline LHS-FFS (100 samples) & 1196.775 & 1214.366 & 1216.895 & 1269.547 & 1278.014 & 1247.022 \\
\hline
\end{tabular}

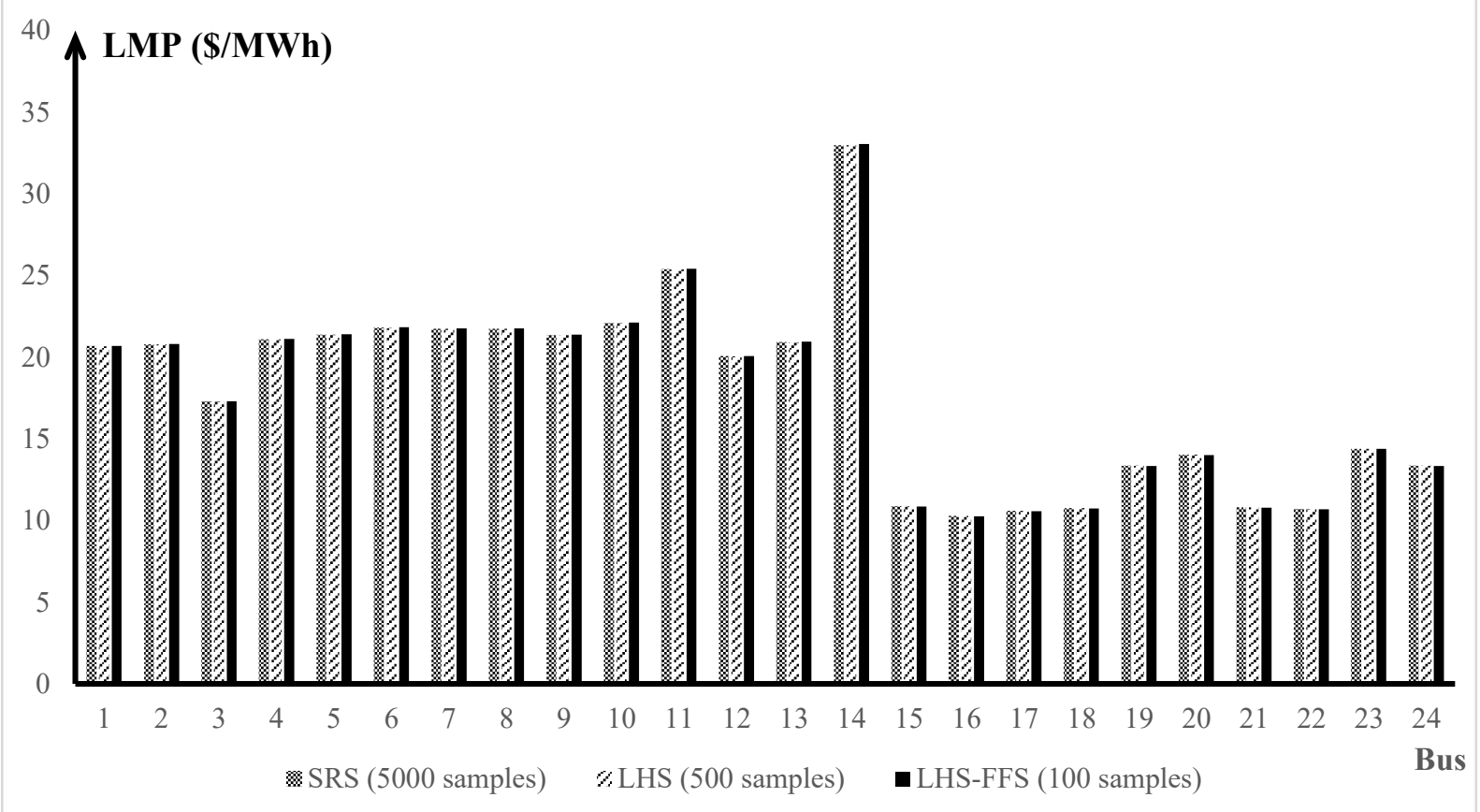

Fig. 5. The mean LMP of IEEE 24-bus system

Using the proposed P-OPF model, the probabilistic locational marginal prices are obtained through Monte Carlo simulation with three different sampling methods, namely simple random sampling (SRS), Latin hypercube sampling (LHS), and Latin hypercube sampling combined with Fast Forward Selection Algorithm (LHS-FFS).

The obtained results that are depicted in Table 4 show that the solution of P-OPF with three sampling techniques is very similar. However, the computational time and computer storage for solving the P-OPF problem with LHS-FFS are considerably reduced.

\subsection{IEEE 24-Bus System}

This subsection presents the calculated results of mean LMP using the IEEE 24-bus system [21]. The diagram of this 24-bus system is revealed in Fig. 4. The resulting LMPs from this system are depicted in Fig. 5.

Fig. 5 shows that the P-OPF problem with three sampling methods all results in identical solutions. On the other hand, there is a significant decrease in the computational time for solving the P-OPF with LHS-FFS (around 5 seconds with 100 samples), compared to approximately 4 minutes when applying SRS (5000 samples). 


\section{Conclusion}

This paper presents methods for calculating probabilistic locational marginal price (LMP) using Monte Carlo Simulation integrated with different sampling techniques. Three sampling approaches are compared, including simple random sampling (SRS), Latin hypercube sampling (LHS), and LHS combined with the fast forward selection (FFS) algorithm to reduce the number of samples. The results show that the mean LMP obtained with these sampling procedures is very similar; however, the computational performance such as time and computer storage is markedly improved when applying LHS-FFS. This contributes to the efficient operation of electricity markets.

\section{References}

[1] Z. Zhao and $\mathrm{L}$. Wu, Impacts of high penetration wind generation and demand response on LMPs in dayahead market, IEEE Trans. Smart Grid, 5 (2014) 220 229.

https://doi.org/10.1109/TSG.2013.2274159

[2] S. Liu, L. Trinh, J. Zhu, and M. Moore, Comprehensive wind power interconnection evaluation method based on LMP market simulation, 1st IEEE-PES/IAS Conf. Sustain. Altern. Energy, SAE 2009 - Proc., 2009.

https://doi.org/10.1109/SAE.2009.5534877

[3] F. Li, S. Member, R. Bo, and S. Member, DCOPFBased LMP Simulation: Algorithm, comparison with ACOPF and sensitivity, IEEE Trans. Power Syst., 22 (2007) 1475-1485.

https://doi.org/10.1109/TPWRS.2007.907924

[4] X. Fang, Y. Wei, and F. Li, Evaluation of LMP Intervals Considering Wind Uncertainty, IEEE Trans. Power Syst., 31 (2016) 2495-2496.

https://doi.org/10.1109/TPWRS.2015.2449755

[5] H. Yu, C. Y. Chung, K. P. Wong, H. W. Lee, and J. H. Zhang, Probabilistic load flow evaluation with hybrid Latin hypercube sampling and Cholesky decomposition, IEEE Trans. Power Syst., 24 (2009) 661-667.

https://doi.org/10.1109/TPWRS.2009.2016589

[6] M. Hajian, W. D. Rosehart, and H. Zareipour, Probabilistic power flow by Monte Carlo simulation with Latin supercube sampling, IEEE Trans. Power Syst., 28 (2013)1550-1559.

https://doi.org/10.1109/TPWRS.2012.2214447

[7] A. Soroudi, M. Ehsan, R. Caire, and N. Hadjsaid, Possibilistic evaluation of distributed generations impacts on distribution networks, IEEE Trans. Power Syst., 26 (2011) 2293-2301.

https://doi.org/10.1109/TPWRS.2011.2116810

[8] A. Soroudi, Possibilistic-scenario model for DG impact assessment on distribution networks in an uncertain environment, IEEE Trans. Power Syst., 27 (2012) 1283-1293.

https://doi.org/10.1109/TPWRS.2011.2180933
[9] A. Soroudi and M. Ehsan, IGDT based robust decision-making tool for DNOs in load procurement under severe uncertainty, IEEE Trans. Smart Grid, 4 (2013) 886-895.

https://doi.org/10.1109/TSG.2012.2214071

[10] N. B. Hargreaves, S. M. Pantea, and G. A. Taylor, Large Scale Renewable Power Generation (Advances in Technologies for Generation, Transmission, and Storage), 2014

[11] J. M. Morales, S. Pineda, A. J. Conejo, and M. Carrion, Scenario reduction for futures market trading in electricity markets, IEEE Trans. Power Syst., 24 (2009) 878-888.

[12] K. C. Sharma, P. Jain, and R. Bhakar, Wind Power Scenario Generation and Reduction in Stochastic Programming Framework, Electr. Power Components Syst., 41 (2013) 271-285.

https://doi.org/10.1109/TPWRS.2009.2016072

[13] Y. Wang, Y. Liu, and D. S. Kirschen, Scenario Reduction with Submodular Optimization, IEEE Trans. Power Syst., 32(2016) 2479-2480. https://doi.org/10.1109/TPWRS.2016.2603448

[14] https://www.mathworks.com/products/matlab.html.

[15] N. Gröwe-Kuska, H. Heitsch, and W. Römisch, Scenario reduction and scenario tree construction for power management problems, 2003 IEEE Bol. PowerTech - Conf. Proc., 3 (2003) 152-158.

[16] H. Heitsch and W. Römisch, Scenario reduction algorithms in stochastic programming, Comput. Optim. Appl., 24 (2003) 187-206.

[17] H. W. Dommel and W. F. Tinney, Optimal Power Flow Solutions, IEEE Trans. Power Appar. Syst., PAS-87 (1968) 1866-1876. https://doi.org/10.1109/TPAS.1968.292150

[18] D. I. Sun, B. Ashley, B. Brewer, A. Hughes, and W. F. Tinney, Optimal power flow by Newton approach, IEEE Trans. Power Appar. Syst., PAS-103 (1984) 2864-2880. https://doi.org/10.1109/TPAS.1984.318284

[19] O. Alsaç, J. Bright, M. Prais, and B. Stott, Further developments in lp-based optimal power flow, IEEE Trans. Power Syst., 5 (1990) 697-711. https://doi.org/10.1109/59.65896

[20] L. S. Vargas, V. H. Quintana, and A. Vannelli, A tutorial description of an interior point method and its applications to security-constrained economic dispatch, IEEE Trans. Power Syst., 8 (1993) 13151324.

https://doi.org/10.1109/59.260862

[21] J. M. Morales and J. Pérez-ruiz, Simulating the Impact of Wind Production on Locational Marginal Prices, IEEE Trans. Power Syst., 26 (2011) 820-828. https://doi.org/10.1109/TPWRS.2010.2052374

[22] https://www.powerworld.com/ 